\title{
Produção e avaliação de microfibras de celulose para aplicação em filmes de amido de mandioca produzidos por tape-casting
}

\author{
R. da SILVA SIMÃO ${ }^{1}$, P. P. de ALMEIDA ${ }^{1}$, N. B. PASCUAL $^{1}$ e J. B. LAURINDO ${ }^{1}$ \\ ${ }^{1}$ Universidade Federal de Santa Catarina, Departamento de Engenharia Química e \\ Engenharia de Alimentos \\ E-mail para contato: raquelsimao@hotmail.com
}

\begin{abstract}
RESUMO - Fibras de celulose de eucalipto foram submetidas a tratamentos ácido e mecânico em ultrassom para produção de micropartículas. De acordo com o seu tamanho e estabilidade, selecionaram-se duas microfibras de celulose para adição em filmes de amido: F1 - obtida por tratamento mecânico em ultrassom (tamanho $\leq 600 \mu \mathrm{m}$ ); e F2- obtida por tratamento ácido + mecânico em ultrassom (tamanho $\leq 50 \mu \mathrm{m}$ ). Para avaliação do efeito das microfibras de celulose nas propriedades de filmes de amido de mandioca produzidos por tape-casting, três formulações de suspensão filmogênica foram testadas: $\mathrm{A} 0=5 \mathrm{~g}$ amido/ $100 \mathrm{~mL}$ de água $+20 \mathrm{~g}$ glicerol $/ 100 \mathrm{~g}$ amido; $\mathrm{A} 1=\mathrm{A} 0+0,1 \mathrm{~g} \mathrm{~F} 1 / 100 \mathrm{~mL}$ água; e A2 $=5 \mathrm{~g}$ amido/100 $\mathrm{mL}$ água $+30 \mathrm{~g}$ glicerol/ $100 \mathrm{~g}$ amido $+0,2 \mathrm{~g} \mathrm{~F} / 100 \mathrm{~mL}$ água. A adição de $\mathrm{F} 1$ reduziu à metade a solubilidades dos filmes em relação ao filme $\mathrm{A} 0$, sendo esta ainda menor com a adição de F2. A adição de F1 e F2 influenciaram as propriedades mecânicas dos filmes, pelo aumento da sua capacidade de alongamento em 30 vezes, apesar da redução nos valores de Tensão e Módulo de Elasticidade.
\end{abstract}

\section{INTRODUÇÃO}

Os filmes a base de amido apresentam características (biodegradáveis, transparentes e flexíveis) que os tornam interessantes para aplicação em embalagens de alimentos. Eles apresentam baixa permeabilidade a gases, porém possuem propriedades mecânicas e de barreira ao vapor de água inferiores às dos plásticos sintéticos. Sua higroscopicidade é a principal desvantagem associada ao seu uso, tornando necessária a melhora da sua resistência (Müller et al., 2009).

A fim de suprir essas deficiências, os filmes de amido têm sido adicionados de compostos, tais como fibras de celulose, que apresentam grande compatibilidade com a matriz polimérica de amido, como resultado de interações intermoleculares entre os componentes (Wollerdorfer e Bader, 1998; Curvelo et al., 2001; Follain et al., 2006; Müller, 2007). Os tratamentos de fibras de celulose permitem a produção de micropartículas capazes de se integrar de maneira mais eficiente em estruturas poliméricas. Os tratamentos mais comuns incluem a hidrólise ácida/alcalina, que facilita a quebra das fibras originais em celulose microcristalina (Lu, Weng e Cao, 2005); e o tratamento mecânico em ultrassom, que produz oscilação devido à cavitação, e forças hidrodinâmicas para separar as fibras celulósicas em micro/nano escalas (Wang e Cheng, 2009). Vários estudos afirmam que o tamanho e a concentração de fibras na suspensão filmogênica podem levar a resultados diferentes. 
Filmes a base de amido necessitam da adição de plastificantes, como o glicerol, capazes de reduzir as forças intermoleculares, aumentar a mobilidade das cadeias dos polímeros e a flexibilidade dos filmes (Standing et al., 2001; Mali et al., 2010).

O desenvolvimento de filmes pela técnica de tape-casting possibilita uma escala de produção industrial. Nesse processo, a suspensão filmogênica é colocada em um reservatório acoplado a uma lâmina niveladora, com abertura ajustada com parafusos micrométricos. A solução filmogênica é espalhada em uma camada fina sobre um suporte, devido ao movimento da lâmina (processo descontínuo). O filme formado é seco sobre o suporte por condução de calor e circulação de ar quente (convecção de calor) (Moraes et al., 2013).

Diante disso, o objetivo deste trabalho foi avaliar o efeito da adição de microfibras de celulose obtidas com diferentes processos de redução de tamanho, sobre as propriedades de filmes de amido de mandioca produzidos por tape-casting.

\section{MATERIAIS E MÉTODOS}

\subsection{Materiais}

O amido de mandioca foi fornecido pela Companhia Lorenz (Indaial, Santa Catarina), contendo 17 a $20 \%$ de amilose e 80 a $83 \%$ de amilopectina. As fibras utilizadas foram fibras branqueadas de celulose de eucalipto, cedidas pela empresa CENIBRA S/A (Belo Horizonte, Minas Gerais). O glicerol de grau analítico e o ácido clorídrico foram fornecidos pela Vetec Química Fina (Distrito Industrial de Duque de Caxias, Rio de Janeiro).

\subsection{Tratamentos para Redução do Tamanho das Fibras de Celulose}

A Tabela 1 apresenta os métodos utilizados para tratamentos de fibras de celulose.

Tabela 1 - Condições de tratamento de fibras de eucalipto.

\begin{tabular}{|c|c|c|c|}
\hline $\begin{array}{c}\text { Concentração das } \\
\text { Suspensões Filmogênicas } \\
(\mathrm{g} \text { fibras } / 100 \mathrm{~mL})\end{array}$ & Pré-Tratamento $(72 \mathrm{~h})$ & $\begin{array}{c}\text { Hidrólise Ácida } \\
\left(80^{\circ} \mathrm{C}\right)(\mathrm{min})\end{array}$ & $\begin{array}{c}\text { Tratamento em } \\
\text { Ultrassom }(\mathrm{min})\end{array}$ \\
\hline \multirow{3}{*}{0,1} & Água destilada & - & $30,60,90,120$ \\
\cline { 2 - 3 } & Ácido sulfúrico 0,1M & 90 & $30,60,90$ \\
\cline { 2 - 3 } & \multirow{3}{*}{ Ácido clorídrico 2M } & 30 & 30,60 \\
& & $60,90,120$ & \multirow{2}{*}{30} \\
\cline { 3 - 3 } 0,2 & & $30,60,90$ & \\
\cline { 3 - 3 } 0,5 & & &
\end{tabular}

A hidrólise foi realizada sob agitação constante a $80{ }^{\circ} \mathrm{C}$, em banho ultratermostático microprocessado com circulador (QUIMIS, Diadema, São Paulo). E para o tratamento de ultrassom, utilizou-se o ultrassom VCX 500 (Sonics \& Materials, Inc., Newtown, EUA), com pulso de 59 s on e $10 \mathrm{~s}$ off e amplitude de $35 \%$.

\subsection{Caracterização das microfibras de celulose}


A caracterização das microfibras de celulose foi realizada mediantes análises de tamanho de partícula, morfologia e estabilidade (potencial zeta).

O tamanho das fibras foi determinado por difração de laser em Mastersizer 2000 (Malvern Instruments Ltd, Reino Unido), e a morfologia foi analisada em Microscópio Ótico Leica DM4000M, câmera DC300 (Leica Microsystems, Alemanha).

A estabilidade da suspensão de microfibras de celulose foi avaliada pelo potencial zeta, através da medição da diferença de potencial elétrico entre o meio de dispersão e a fase estacionária de fluido ligado às microfibras dispersas em Zetasizer nano ZS (Malven Instruments Ltd, Reino Unido).

\subsection{Elaboração dos filmes de amido e amido/microfibras de celulose por tape-casting}

A Tabela 2 apresenta as formulações de suspensões de amido-glicerol e amido-glicerolfibras testadas.

Tabela 2 - Formulações de filmes testadas.

\begin{tabular}{|c|c|c|c|}
\hline Filme & g amido/ $100 \mathrm{~mL}$ água & g glicerol/100 g amido & g microfibras/100 $\mathrm{mL}$ água \\
\hline 1 & 5 & 20 & 0 \\
\hline 2 & 5 & 22 & 0 \\
\hline 3 & 5 & 23 & 0 \\
\hline 4 & 5 & 24 & 0 \\
\hline 5 & 5 & 25 & 0 \\
\hline 6 & 5 & 30 & 0,1 \\
\hline 7 & 3 & 15 & 0,1 \\
\hline 8 & 4 & 15 & 0,1 \\
\hline 9 & 5 & 15 & 0,1 \\
\hline 10 & 5 & 20 & 0,1 \\
\hline 11 & 5 & 25 & 0,1 \\
\hline 12 & 5 & 30 & 0,2 \\
\hline 14 & 5 & 30 & \\
\hline
\end{tabular}

As condições de processo do equipamento de tape-casting foram fixadas em: temperatura de espalhamento da suspensão filmogênica a $50{ }^{\circ} \mathrm{C}$; abertura da lâmina niveladora em $3 \mathrm{~mm}$, e velocidade de espalhamento da suspensão de 1,8 cm/s. A secagem dos filmes foi realizada sobre o próprio suporte de espalhamento, com o auxílio de um túnel de secagem. Os filmes resultantes foram condicionados a temperatura ambiente $\left(21 \pm 1{ }^{\circ} \mathrm{C}\right)$ e umidade relativa de $58 \%$ durante $2-4$ dias antes da sua caracterização.

\subsection{Caracterização dos Filmes de Amido}

A espessura dos filmes foi determinada em pontos aleatórios de cada amostra, utilizando-se um micrômetro digital MDC-Lite (Mitutoyo Corporation, Japão). 
O teor de umidade dos filmes foi determinado em, no mínimo, triplicata, por secagem das amostras com aproximadamente $0,5 \mathrm{~g}$, em estufa a $105^{\circ} \mathrm{C}$ por $24 \mathrm{~h}$, de acordo com Association of Official Analytical Chemists (A.O.A.C., 1995).

Determinou-se a solubilidade dos filmes em água conforme proposto em Núñez-Flores et al. (2012). Todas as análises foram realizadas em triplicata.

Os testes de tração foram realizados em Texturômetro TA-XT2i (Stable Micro System, Surrey, Reino Unido), conforme proposto em Núñez-Flores et al. (2012). Todas as análises foram realizadas em triplicata.

\section{RESULTADOS E DISCUSSÃO}

\subsection{Microfibras de Celulose}

As fibras apresentaram uma distribuição de tamanho de $d_{10}, d_{50}$ e $d_{90}$, variando entre 6,916,$4 ; 17,5-40,6$ e $110,4-790,4 \mu \mathrm{m}$, respectivamente, confirmando a eficácia dos tratamentos para a produção de microfibras. Tratamentos de $\mathrm{HCl}+$ ultrassom resultaram em tamanhos menores $\left(\mathrm{d}_{90}=40,63-77,76 \mu \mathrm{m}\right)$, enquanto os tratamentos de $\mathrm{H}_{2} \mathrm{O}+$ ultrassom e $\mathrm{H}_{2} \mathrm{SO}_{4}+$ ultrassom resultaram em maiores tamanhos $\left(\mathrm{d}_{90}=589.60-790,42 \mu \mathrm{m}\right)$. O tempo de tratamento de ultrassom reduziu proporcionalmente o tamanho da fibra em $\mathrm{H}_{2} \mathrm{O}$, e não afetou as tratadas com $\mathrm{H}_{2} \mathrm{SO}_{4}$ (Figura 1).

Figura 1 - Tamanhos $(\mu \mathrm{m})$ de microfibras produzidas a partir de diferentes tempos $(\mathrm{min})$ de tratamentos em ultrassom $\left(\mathrm{H}_{2} \mathrm{SO}_{4}+\right.$ ultrassom e $\mathrm{HCl}+$ ultrassom com hidrólise de $\left.90 \mathrm{~min}\right)$.

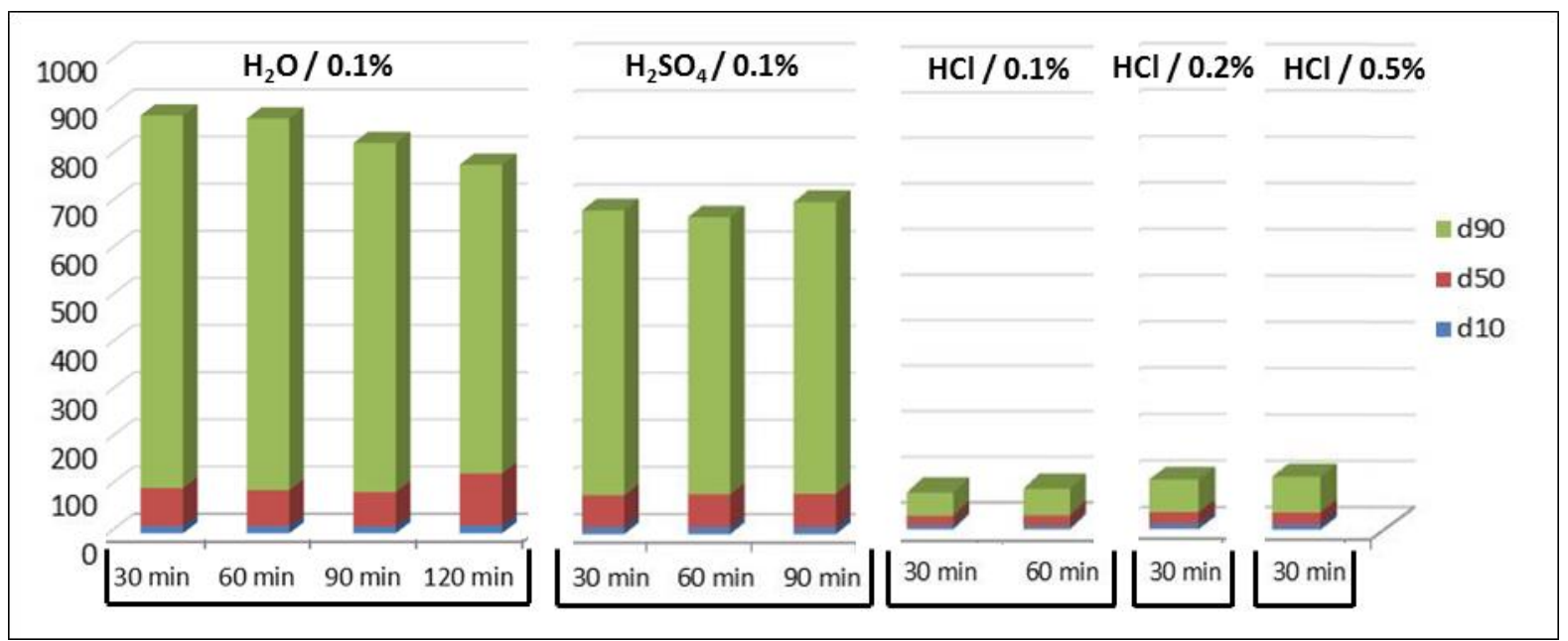

O potencial zeta (Tabela 3) pode ser utilizado como uma medida das forças eletrostáticas repulsivas e indicação da estabilidade da suspensão de fibras durante o desenvolvimento dos filmes. Enquanto o valor do potencial zeta das fibras sem tratamento foi de $-5,27 \pm 0,11 \mathrm{mV}$, os valores de potencial zeta após os tratamentos variaram entre -13,80 e $37,7 \mathrm{mV}$. Um valor absoluto menor ou maior do que $25 \mathrm{mV}$ é indicativo da capacidade de floculação das fibras ou não, respectivamente. Apesar do tratamento com $\mathrm{H}_{2} \mathrm{SO}_{4}+$ ultrassom reduzir ligeiramente o tamanho das fibras em comparação com o tratamento de $\mathrm{H}_{2} \mathrm{O}+$ ultrassom, os resultados dos potenciais zeta de $\mathrm{H}_{2} \mathrm{SO}_{4}+$ ultrassom $(-13,80$ a $-18,84 \mathrm{mV})$ 
indicaram que este não produziu suspensões estáveis, enquanto que o tratamento de $\mathrm{H}_{2} \mathrm{O}+$ ultrassom $(-28,77 \mathrm{a}-32,25 \mathrm{mV})$ resultou em suspensões estáveis. Uma vez que o tempo de ultrassom (30 e $60 \mathrm{~min}$ ) não afetou negativamente o potencial zeta das fibras tratadas com $\mathrm{HCl}+$ ultrassom $(-33,47$ e $-37,57 \mathrm{mV}$, respectivamente), nem o tamanho da fibra $(45,08 \mathrm{e}$ $58,16 \mu \mathrm{m}$, respectivamente), tempos de hidrólise e concentrações de fibras maiores foram testados.

Tabela 3 - Potencial zeta das microfibras obtidas com diferentes tratamentos.

\begin{tabular}{|c|c|c|c|c|c|}
\hline Amostra & $\begin{array}{c}\mathrm{g} \text { fibras } / 100 \mathrm{~mL} \\
\text { água }\end{array}$ & $\begin{array}{l}\text { Pré-tratamento } \\
(72 \mathrm{~h})\end{array}$ & $\begin{array}{l}\text { Hidrólise } \\
80^{\circ} \mathrm{C} \text { (min) }\end{array}$ & $\begin{array}{c}\text { Tratamento } \\
\text { ultrassom (min) }\end{array}$ & $\begin{array}{l}\text { Potencial zeta } \\
(\mathrm{mV})\end{array}$ \\
\hline 0 & 0,5 & \multirow{5}{*}{ Água destilada } & \multirow{5}{*}{ NO } & $\mathrm{NO}$ & $-5,27 \pm 0,11$ \\
\hline 1 & \multirow{4}{*}{0,1} & & & 30 & $-32,05 \pm 0,21$ \\
\hline 2 & & & & 60 & $-31,70 \pm 0,05$ \\
\hline 3 & & & & 90 & $-32,25 \pm 1,06$ \\
\hline 4 & & & & 120 & $-28,77 \pm 1,25$ \\
\hline 5 & \multirow{3}{*}{0,1} & \multirow{3}{*}{$\begin{array}{c}\text { Ácido sulfúrico } \\
0,1 \mathrm{M}\end{array}$} & \multirow{3}{*}{90} & 30 & $-13,80 \pm 0,71$ \\
\hline 6 & & & & 60 & $-15,90 \pm 0,99$ \\
\hline 7 & & & & 90 & $-18,84 \pm 1,22$ \\
\hline 8 & \multirow{5}{*}{0,1} & \multirow{11}{*}{$\begin{array}{c}\text { Ácido clorídrico } \\
2 \mathrm{M}\end{array}$} & 30 & \multirow{4}{*}{30} & $-30,87 \pm 1,29$ \\
\hline 9 & & & 60 & & $-29,20 \pm 0,36$ \\
\hline 10 & & & 90 & & $-27,70 \pm 0,56$ \\
\hline 11 & & & & & $-33,47 \pm 0,32$ \\
\hline 12 & & & 120 & 60 & $-37,67 \pm 1,01$ \\
\hline 13 & \multirow{3}{*}{0,2} & & 30 & \multirow{6}{*}{30} & $-28,07 \pm 1,06$ \\
\hline 14 & & & 60 & & $-28,97 \pm 0,25$ \\
\hline 15 & & & 90 & & $-30,87 \pm 0,90$ \\
\hline 16 & \multirow{3}{*}{0,5} & & 30 & & $-18,90 \pm 0,26$ \\
\hline 17 & & & 60 & & $-30,83 \pm 0,64$ \\
\hline 18 & & & 90 & & $-27,47 \pm 0,15$ \\
\hline
\end{tabular}

Todos os tratamentos de $\mathrm{HCl}+$ ultrassom com concentrações de fibras menores $(0,1 \mathrm{e}$ $0,2 \%$ ) resultaram em suspensões estáveis $(-27,70 \mathrm{a}-37,67 \mathrm{mV})$, enquanto que a concentração mais elevada de fibra $(0,5 \%)$ necessitou de tempos de hidrólise maiores ou iguais a 60 minutos para se obter suspensões estáveis.

A microscopia ótica confirmou os tamanhos de fibras de cada tratamento e mostrou que os tratamentos com $\mathrm{H}_{2} \mathrm{O}+$ ultrassom e $\mathrm{H}_{2} \mathrm{SO}_{4}+$ ultrassom resultaram em microfibras maiores e cilíndricas, enquanto que nos tratamentos com $\mathrm{HCl}+$ ultrassom, as fibras apresentaram-se sob a forma de fragmentos pequenos com tendência a se agregar em pequenos grupos (Figura 2).

Diante disso, duas microfibras foram selecionadas para o desenvolvimento de filmes de amido com celulose: $\mathrm{F} 1=0,1 \%$ e tratamento em $\mathrm{H}_{2} \mathrm{O}+$ ultrassom por 90 min; e $\mathrm{F} 2=0,5 \%$ e tratamento em $\mathrm{HCl} 60 \mathrm{~min}+$ ultrassom $30 \mathrm{~min}$. 
Figura 2 - Efeito dos tratamentos sobre a morfologia e o tamanho das fibras (Letras "a", "b", "c" e "d" referem-se aos tratamentos com ácidos nas mesmas condições: 90 min de hidrólise e 30 min de tratamento ultrassônico; letra "e" refere-se ao tratamento de $\mathrm{H}_{2} \mathrm{O}$ e 30 minutos de tratamento com ultrassom e a letra " $\mathrm{f}$ " as fibras de celulose sem tratamento).

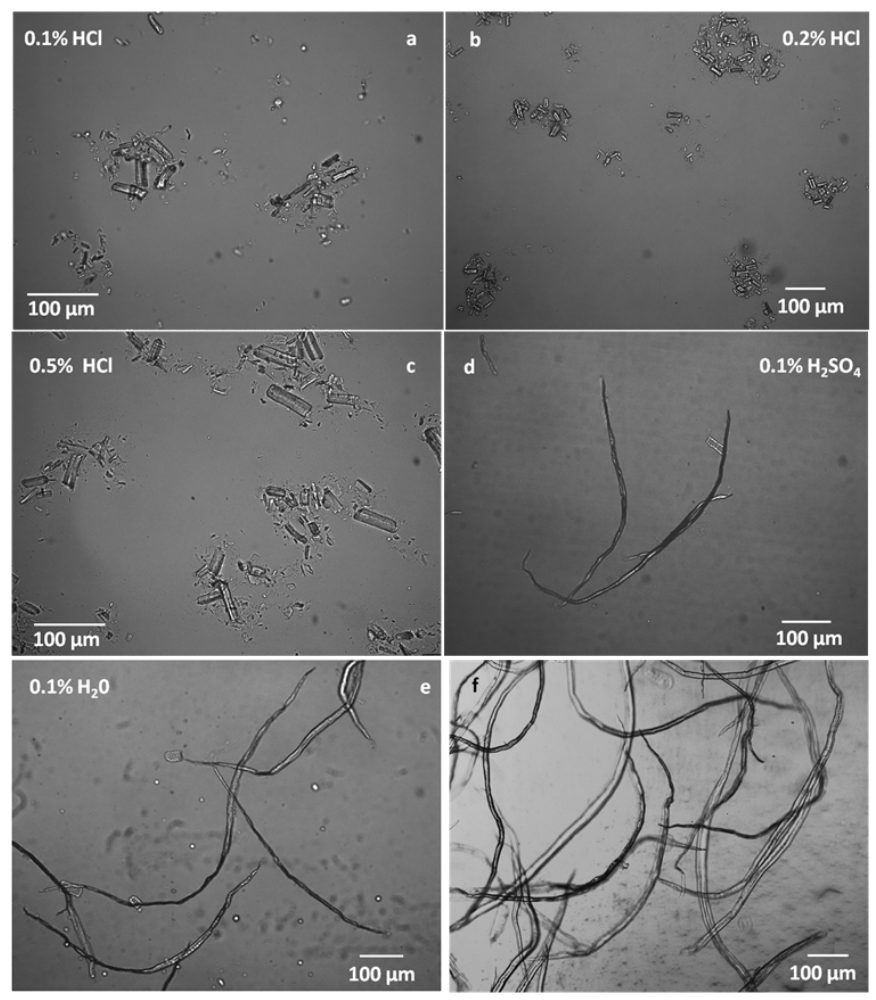

\subsection{Filmes de Amido}

As formulações com 3 e 4\% de amido (Tabela 4) resultaram em suspensões pouco viscosas e difíceis de serem espalhadas. Com $15 \%$ de glicerol, os filmes resultantes apresentaram-se quebradiços, bem como os filmes com concentrações de glicerol de $25 \%$ ou menores, e os filmes com adição das F2 foram quebradiços. Com 30\% de glicerol, os filmes de amido sem fibras e os filmes com adição das F1 e F2 0,1\% resultaram excessivamente pegajosos. Assim, as formulações que resultaram em melhores filmes foram: $5 \%$ de amido e $20 \%$ de glicerol para os filmes de amido sem fibras e com adição das F1 $(0,1 \%)$, e $30 \%$ de glicerol para os filmes com adição das F2 (0,2\%). 
Tabela 4 - Formulações de filmes testadas

\begin{tabular}{|c|c|c|c|c|c|}
\hline Filme & $\begin{array}{c}\text { g amido/100 } \\
\text { mL água }\end{array}$ & $\begin{array}{c}\text { g glicerol/ } \\
100 \mathrm{~g} \text { amido }\end{array}$ & $\begin{array}{c}\mathrm{g} \mathrm{F} 1 / 100 \mathrm{~mL} \\
\text { água }\end{array}$ & $\begin{array}{c}\mathrm{g} \mathrm{F} 2 / 100 \mathrm{~mL} \\
\text { água }\end{array}$ & Espessura $(\mu \mathrm{m})$ \\
\hline 1 & 5 & 20 & 0 & 0 & $94,14 \pm 14,06$ \\
\hline 3 & 5 & 23 & 0 & 0 & $111,50 \pm 5,80$ \\
\hline 4 & 5 & 24 & 0 & 0 & $94,50 \pm 13,03$ \\
\hline 5 & 5 & 25 & 0 & 0 & $100,76 \pm 8,69$ \\
\hline 6 & 3 & 15 & 0,1 & 0 & $82,8 \pm 9,1$ \\
\hline 7 & 4 & 15 & 0,1 & 0 & $86,0 \pm 4,8$ \\
\hline 8 & 5 & 15 & 0,1 & 0 & $104,7 \pm 4,7$ \\
\hline 9 & 5 & 20 & 0,1 & 0 & $116,0 \pm 3,9$ \\
\hline 10 & 5 & 30 & 0,1 & 0 & $149,4 \pm 5,4$ \\
\hline 11 & 5 & 20 & 0 & 0,1 & $105,38 \pm 6,65$ \\
\hline 12 & 5 & 25 & 0 & 0,1 & $117,60 \pm 8,68$ \\
\hline 13 & 5 & 30 & 0 & 0,1 & $98,00 \pm 12,06$ \\
\hline 15 & 5 & 30 & 0 & 0.2 & $99,41 \pm 11,25$ \\
\hline
\end{tabular}

A Tabela 5 apresenta as propriedades físico-químicas, mecânicas e de barreira de água.

Tabela 5 - Espessura (E), Umidade (U), Solubilidade (S), Tensão (T), Alongamento (E) e Módulo de elasticidade Young (Y) dos filmes (A) desenvolvidos com $5 \mathrm{~g}$ amido/100 $\mathrm{mL}$ água $+20 \mathrm{~g}$ glicerol/100 $\mathrm{g}$ amido (A0), F0 + 0,1 g F1/100 mL água (A1) e $5 \mathrm{~g}$ amido/100 $\mathrm{mL}$ água $+30 \mathrm{~g}$ glicerol/100 g amido $+0,2 \mathrm{~g} \mathrm{~F} / 100 \mathrm{~mL}$ água (A2).

\begin{tabular}{ccccccc}
\hline A & $\mathrm{E}(\mu \mathrm{m})$ & $\mathrm{U}(\%)$ & $\mathrm{S}(\%)$ & $\mathrm{T}(\mathrm{MPa})$ & $\mathrm{E}(\%)$ & $\mathrm{Y}(\mathrm{MPa})$ \\
\hline A0 & $94,14 \pm 14,06^{\mathrm{a}}$ & $10.66 \pm 0.16^{\mathrm{a}}$ & $21.40 \pm 5.89^{\mathrm{a}}$ & $15.78 \pm 4.18^{\mathrm{a}}$ & $2.49 \pm 0.64^{\mathrm{a}}$ & $5.96 \pm 1.02^{\mathrm{a}}$ \\
A1 & $116.0 \pm 3.9^{\mathrm{a}}$ & $10.46 \pm 0.25^{\mathrm{a}}$ & $8.93 \pm 2.13^{\mathrm{b}}$ & $6.04 \pm 0.91^{\mathrm{b}}$ & $60.34 \pm 8.80^{\mathrm{b}}$ & $0.97 \pm 0.19^{\mathrm{b}}$ \\
A2 & $99.41 \pm 11.25^{\mathrm{a}}$ & $17.28 \pm 0.50^{\mathrm{b}}$ & $6.71 \pm 0.34^{\mathrm{c}}$ & $2.85 \pm 0.09^{\mathrm{c}}$ & $63.97 \pm 16.10^{\mathrm{b}}$ & $0.92 \pm 0.08^{\mathrm{b}}$ \\
\hline
\end{tabular}

Os resultados são a média \pm desvio padrão. ANOVA: Letras diferentes na mesma coluna indicam diferenças significativas entre os diferentes filmes $(p \leq 0,05)$.

Não houve diferenças significativas $(\mathrm{p} \leq 0,05)$ na espessura dos filmes desenvolvidos, mas o filme A2 apresentou um teor de umidade maior, devido à maior quantidade de glicerol utilizada. Por outro lado, a adição de F1 de maior tamanho diminuiu significativamente $(\mathrm{p} \leq 0,05)$ a solubilidades dos filmes (pela metade), sendo esta ainda menor com a adição de F2. Além de diminuir a solubilidade, a adição de F1 e F2 aumentou significativamente $(\mathrm{p} \leq 0,05)$ a capacidade de alongamento dos filmes (30 vezes maior), apesar da Tensão e o Módulo de elasticidade terem reduzido.

Trabalhos prévios têm mostrado que as fibras de celulose podem compensar a natureza hidrofilica dos filmes de amido plastificados, melhorando seu manuseamento e resistência à tração (Müller et al., 2009). No geral, fibras grandes melhoram a resistência à tração, mas também reduzem a capacidade do filme de alongamento. E já foi sugerido que tamanhos menores de fibras ou menores concentrações, como as usadas neste trabalho, melhoram a 


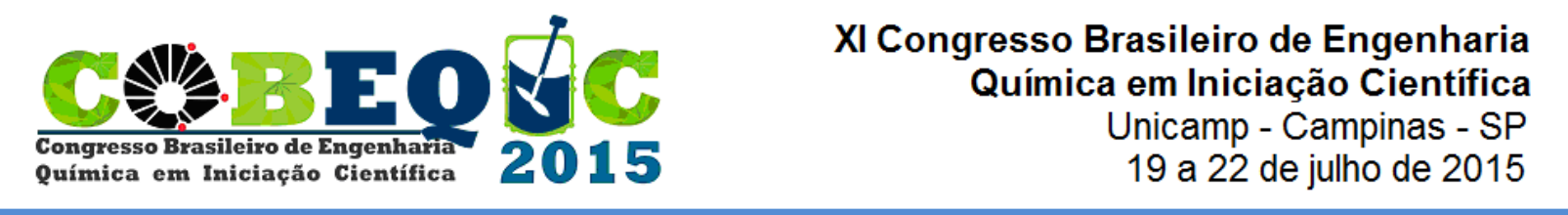

estabilidade do filme em umidade relativa elevada, sem impactar muito nas propriedades mecânicas.

\section{CONCLUSÕES}

A adição de microfibras de celulose de tamanhos em torno 50-600 $\mu \mathrm{m}$ em concentrações baixas $(0,1-0,2 \%)$ melhora consideravelmente a resistência à água e a capacidade de alongamento dos filmes de amido de mandioca, compensando o problema da excessiva rigidez que a celulose normalmente confere a estes filmes.

\section{REFERÊNCIAS}

AOAC. Association of Official Analytical Chemits. Official methods of analysis of the AOAC International. $16^{\mathrm{a}}$ ed. Arlington, AOAC, 1995.

CURVElo, A. A. S.; CARvalho, A. J. F.; AGNElli, J. A. M.; Thermoplastic starch cellulosic fiber composites: Preliminary results. Carbohydr. Polym., 45, 183-188, 2001.

FOLLAIN, N.; JOLY, C.; DOLE, P.; ROGE, B.; MATHLOUTHI, M. Quaternary starch based blends: Influence of fouth component addiction to the starch/water/glycerol systems. Carbohydr. Polym., 63, 400-407, 2006.

LU, Y.; WENG, L.; CAO, X. Biocomposites of plasticized starch reinforced with cellulose crystallites from cottonseed linter. Macromol. Biosci. 5(11), 1101-1107, 2005.

MALI, S., GROSSMANN; M. V. E.; YAMASHITA, F. Filmes de amido: produção, propriedades e potencial de utilização. Semina: Ci. Agrárias, Londrina, 31(1), 137-156, 2010.

MORAES, J. O.; SCHEIBE, A. S., SERENO, A.; LAURINDO, J. B. Scale-up of the production of cassava starch based films using tape-casting. J. Food Eng., 119, 800808, 2013.

MÜLLER, C. M. O., LAURINDO, J. B.; YAMASHITA, F. Effect of cellulose fibers addition on the mechanical properties and water vapor barrier of starch-based films. Food Hydrocolloid., 23, 1328-1333, 2009.

NÚÑEZ-FLORES, R., GIMÉNEZ, B., FERNÁNDEZ-MARTÍN, F., LÓPEZ-CABALLERO, M. E., MONTERO, M. P.; GÓMEZ-GUILLÉN, M. C. Role of lignosulphonate in properties of fish gelatin films. Food Hydrocolloid., 27, 60-71, 2012.

STADING, M.; RINDLAV-WESTLING, A.; GATENHOLM, P. Humidity-induced structural transitions in amylose and amylopectin films. Carbohydr. Polym., 45(3), 209-217, 2001.

WANG, S.; CHENG, Q. A novel process to isolate fibrils from cellulose fibers by high-intensity ultrasonication, Part 1: Process optimization. J. Appl. Polym. Sci., 113(2), 1270-1275, 2009. 
WOLLERDORFER, M.; BADER, H. Influence of natural fibers on the mechanical properties of biodegradable polymers. Ind. Crop. Prod., 8, 105-112, 1998. 